\title{
La resistencia mecánica a compresión como parámetro para el control y seguimiento de la arena normalizada
}

\author{
ARENAS en el IETcc. 2. ${ }^{a}$ Parte
}

GREGORIO TABERA SECO

Ingeniero Técnico Industrial

IETcc/CSIC

Fecha de recepción: $21-111-88$

\section{RESUMEN}

En este trabajo se especifica el procedimiento seguido para el control continuo de la calidad de la "arena normalizada", empleada en los ensayos mecánicos de resistencia de los morteros de cemento.

Con esta finalidad se exponen las diferentes medidas tomadas para el control de los aparatos y utensilios empleados, cuyo objeto es conseguir una homogeneización en la confección y resultados de los ensayos que permitan realizar estudios comparativos fiables con la "arena de referencia".

Al final del trabajo se insertan, para su estudio comparativo, hojas de resultados obtenidos por el Instituto con "arenas normalizadas", fabricadas en la Planta de Preparación de Arenas del mismo.

\section{SUMMARY}

This work presents, the process for the continue quality control of the "standardized sand", wich is used in the resistance mechanic essays in the cement mortars.

With this finality, are exposed the different measures for the control of the apparatus and instruments that have been used to obtain a homogenity in the results of the assays, wich allows realize comparative studies that are reliable wich the "reference sand".

At the end of this work is inserted for its comparative study, the results obtained in the Institute with "standardirez sand", that has been manufactured at the right place of the Institute.

\section{INTRODUCCION}

Desde la iniciación de la fabricación del cemento, su control y evaluación, mediante la determinación de sus resistencias mecánicas, fue parámetro fundamental.

Para ello, al utilizar probetas de mortero fue requisito esencial la invariabilidad de sus componentes agua y arena, quedando como parámetro libre el cemento, cuyas cualidades podían, por tanto, ser deducidas de acuerdo con los resultados obtenidos.

Por lo tanto, para poder controlar la calidad del cemento, era imprescindible disponer de una "arena normalizada", común para todos los ensayos tanto en las fábricas de cemento como en los laboratorios de control.

Las vicisitudes y circunstancias por las que atraviesa la "arena normalizada" hasta 1956, año en que se le encargó al entonces Instituto Técnico de la Construcción y del Cemento, como Organismo Oficial, la elaboración y suministro de "arenas normalizadas", están reflejadas por M. Chinchilla en la 1. ' Parte de este trabajo.

El control de las características químicas, físicas y mecánicas de la "arena normalizada", exigidas en las especificaciones, lo efectúa el Instituto desde el comienzo de su producción.

Durante varios años, el Instituto colabora con el CERILH (Centro de Estudios e Investigación de la Industria del Cemento), participando en los "Ensayos Interlaboratorios", que este Organismo Internacional organiza todos los años entre más de 140 laboratorios de 25 países de todo el Mundo. Por estos intercambios de contraste, entre otros, el Instituto ha podido evaluar la calidad de la "arena normalizada" que 
fabricaba, en relación con las normalizadas en estos países y, particularmente, con la AFNOR francesa y la DIN alemana.

Desde el año 1960, la "arena normalizada" española se ajusta a lo estipulado en el Pliego de Prescripciones Técnicas Generales para la Recepción de Cementos $\mathrm{y}$, posteriormente en 1975, al Pliego RC-75.

Al aprobarse la Norma UNE 80-101-84 ("Métodos de Ensayo de Cementos" Determinación de la Resistencia Mecánica), norma adaptada a la europea CEN-EN-196-1, la "arena normalizada" sufre una serie de modificaciones, que obliga al Instituto a fabricar una nueva "arena" de acuerdo con lo estipulado en dicha Norma UNE y que, a la vez, pueda en un futuro próximo ser considerada como "arena normalizada CEN", ya que se somete a un control análogo al que el Instituto Eduardo Torroja viene realizando sobre la arena empleada en los cementos, referido exclusivamente al procedimiento operatorio para la determinación de las resistencias mecánicas.

\section{CONTROL DEL PROCEDIMIENTO}

Teniendo en cuenta que la repetitibilidad de los resultados de los ensayos de resistencia depende de la consecución de unas condiciones idénticas en su ejecución, es necesario verificar periódicamente el calibrado y ajuste de los apáratos, así como el procedimiento operatorio.

La notable importancia de una calidad contrastada ha obligado siempre, a los técnicos del Instituto, ha efectuar un seguimiento continuado de la arena en su Laboratorio de Ensayos Físicos y Mecánicos de Conglomerantes Hidráulicos, mediante el análisis de resultados obtenidos con cementos definidos y la toma de muestras aleatorias de arena producida en la Planta de Preparación, antes de su envio al usuario.

\subsection{Aparatos}

\subsubsection{Amasadora}

La amasadora (Fig. 1) debe proporcionar una mezcla homogénea, sin romper los granos de arena, para lo cual todos los meses se comprueba el espacio existente entre la pala y el recipiente. El control del tiempo de amasado, cuando es automático, se efectúa cada 15 días. Anualmente se realiza una comprobación general.

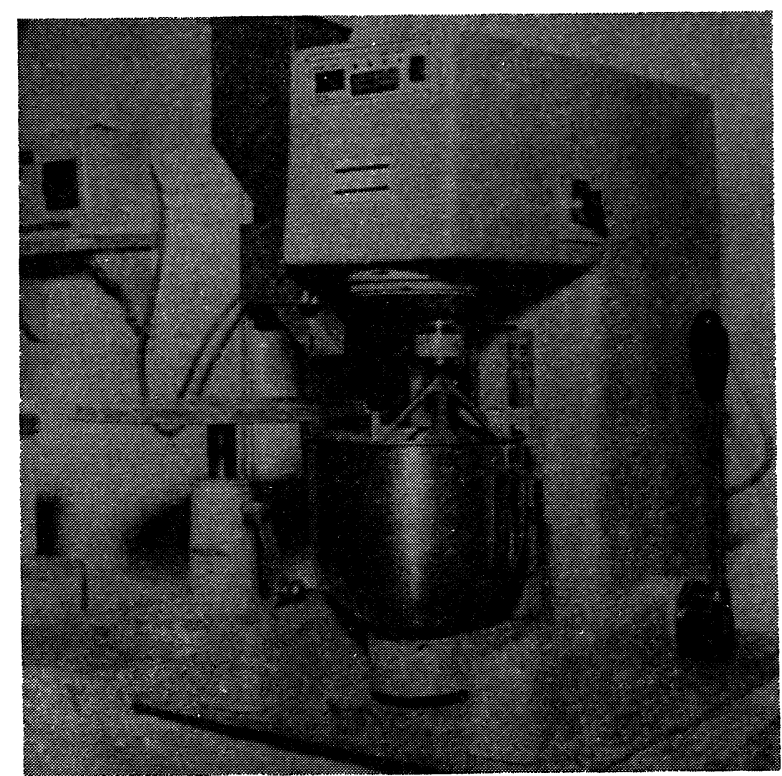

Fig. 1.-Amasadora automática con visor de tiempo digital.

\subsubsection{Compactadora}

Una buena compactación es esencial si se quieren conseguir resultados homogéneos, por tanto es necesario verificar las partes fundamentales de la compactadora (Fig. 2), como son:

- distancia entre la mesa y el eje de giro;

- altura de la caída libre entre yunque y tope;

- desplazamiento horizontal de la mesa;

- comprobación de que las 60 sacudidas tienen lugar en 60 segundos;

- horizontalidad de la mesa menor de $2 \mathrm{~mm}$ de esquina a esquina.

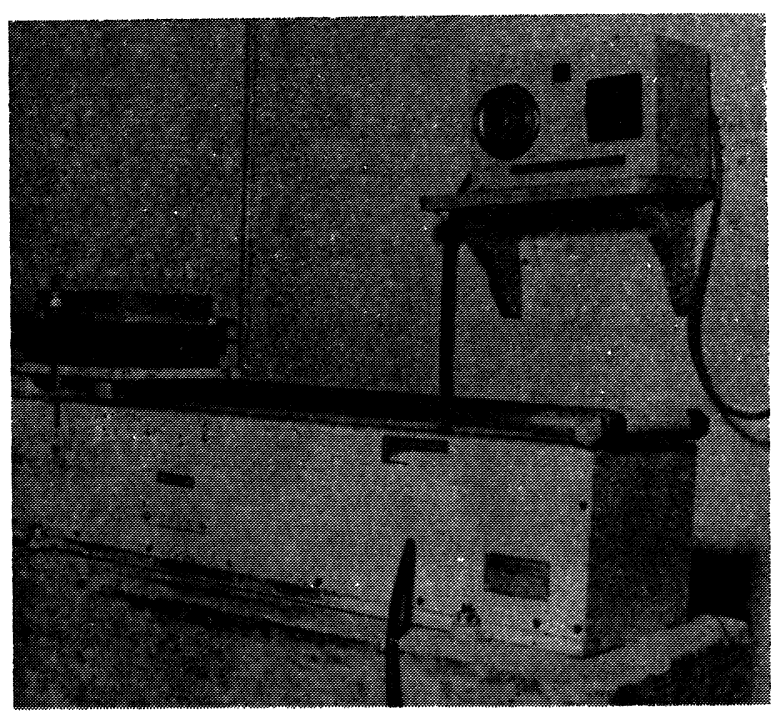

Fig. 2.-Compactadora y contador del número de sacudidas.

MATERIALES DE CONSTRUCCION, Vol. 38, n.²10, abril/mayo/junio 1988 
Todas estas comprobaciones son efectuadas mensual o trimestralmente, según los casos.

\subsubsection{Balanza}

Se utiliza una balanza monoplato que tiene una precisión de $\pm 0,1 \mathrm{~g}$ y es revisada, como mínimo, una vez al año.

\subsubsection{Moldes}

Se emplean moldes de acero resistentes al desgaste y a la corrosión.

Cada tres meses se comprueba su exactitud dimensional e indeformabilidad, mediante galgas e instrumentos apropiados.

Los moldes, antes de ser empleados, son lubricados con una mezcla aceitosa especial que evita se pegue el mortero sobre el acero.

\subsection{Máquinas de Ensayo}

\subsubsection{Flexión}

Para el ensayo de rotura a flexión de las probetas se dispone de un péndulo automático, capaz de medir cargas hasta $10 \mathrm{kN}$ con precisión de $\pm 1 \%$ y una velocidad constante de $50 \mathrm{~N} / \mathrm{s}$. Su revisión se hace anualmente.

\subsubsection{Compresión}

La prensa empleada para el ensayo de compresión (Fig. 3), tiene una capacidad máxima de $20 \mathrm{Mp}$ y dos escalas de $4 \mathrm{Mp}$ y $20 \mathrm{Mp}$, con dispositivo automatico y visor de carga digital en kp. La prensa se debe revisar, tarar y cambiar de aceite una vez al año como mínimo.

\subsection{Materiales}

\subsubsection{Cemento}

El cemento seleccionado para los ensayos de control de la arena se conserva en recipientes metálicos, llenos y herméticamente cerrados hasta su empleo.

El cemento elegido puede ser cualquiera de los tipos existentes en el mercado.

\subsubsection{Arenas}

\subsection{2.a. Arena de referençia}

Con objeto de poder verificar comparativamen-

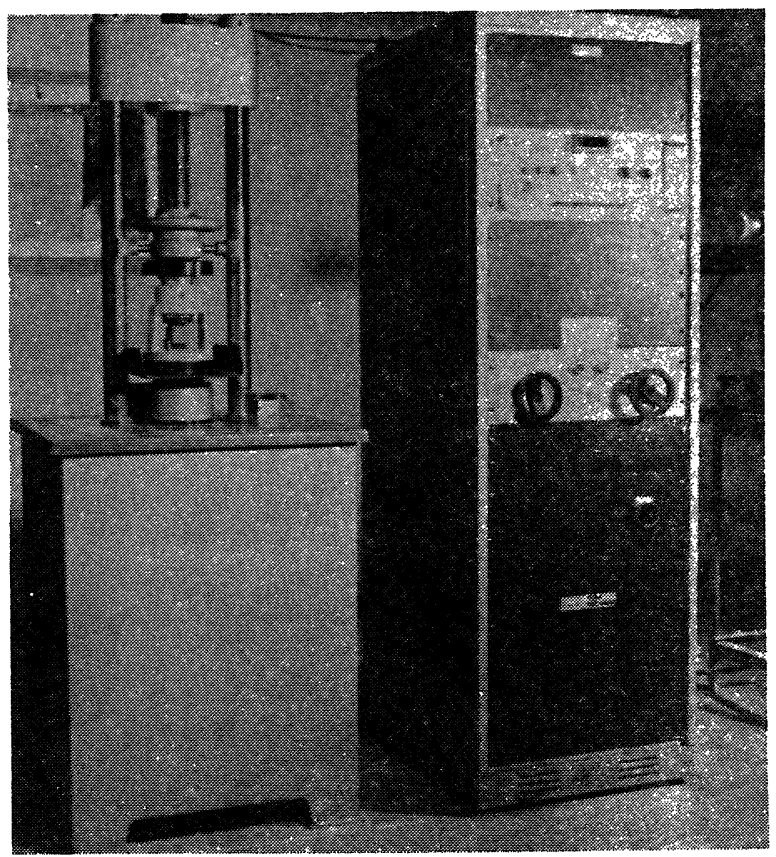

Fig. 3.-Prensa automática de $20 \mathrm{Mp}$, con visor de carga digital.

te el control de calidad de la "arena normalizada", el Instituto ha fabricado expresamente una arena -que se ha designado "arena de referencia" y denominado IET/CEN-, cuyas características son similares a las exigidas en la Norma CEN-EN-196-1 para la "arena normalizada CEN".

\subsection{2.b. Arena normalizada}

La arena normalizada, que se quiere controlar, se selecciona mediante tomas aleatorias obteniéndose en cada toma 3 muestras, cada una correspondiente a la fracción granulométrica de la arena, premezclada, pesada y envasada en una bolsa de plástico hermeticamente cerrada (Fig. 4).

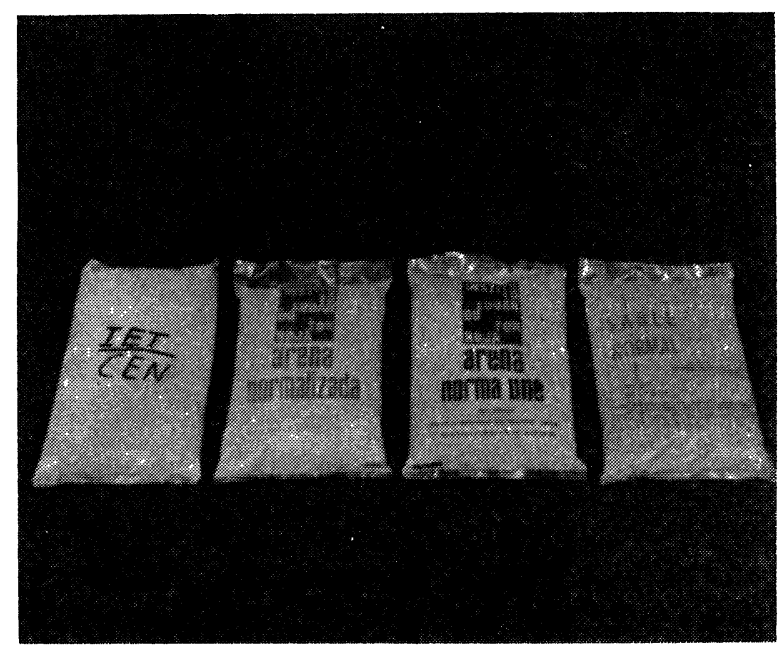

Fig. 4.-Muestras empaquetadas de diferentes arenas normalizadas. 
De cada toma se eligen dos bolsas, quedando la otra en reserva para posibles comprobaciones.

\subsubsection{Agua}

La composición del agua empleada en el amasado del mortero, y en el curado de probetas, es la especificada en el vigente Código Alimentario Español, mantenida a $20^{\circ} \pm 1^{\circ} \mathrm{C}$.

\subsection{Cámara de curado}

Para el curado de probetas se dispone de un local, perfectamente acondicionado y controlado, donde están instaladas las balsas de curado y el armario húmedo. El control de la humedad, $95 \%$, y de la temperatura, $20^{\circ} \pm$ $\pm 1^{\circ} \mathrm{C}$, en el armario se hace mediante dispositivo automático con registrador (Fig. 5). La cámara de curado dispone, para su control de climatización, de un sicómetro, cuyos valores se comprueban diariamente con el fin de mantener una temperatura de $20^{\circ} \pm 1^{\circ} \mathrm{C}$ y una humedad superior al $50 \%$.

Las balsas de curado (Fig. 6) se controlan diariamente manteniendo el agua a $20^{\circ} \pm 1^{\circ} \mathrm{C}$; el cambio parcial del agua se efectúa cada tres meses.

Las probetas, una vez desmoldadas y marcadas, se sumergen inmediatamente en el agua

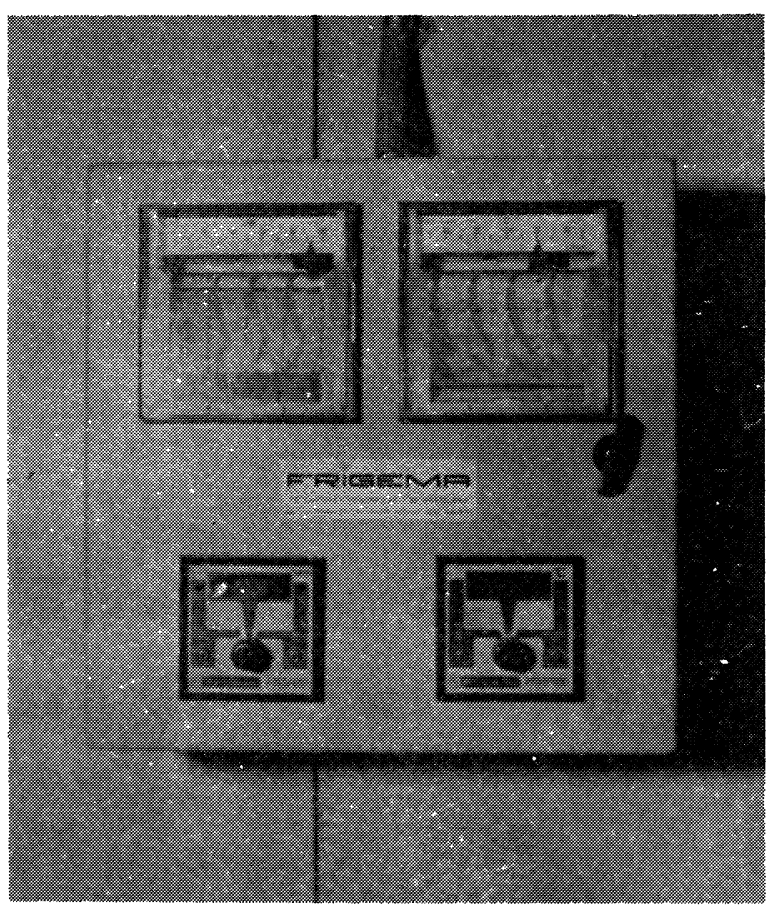

Fig. 5.-Dispositivo automático para el control de humedad y temperatura en la cámara de curado.

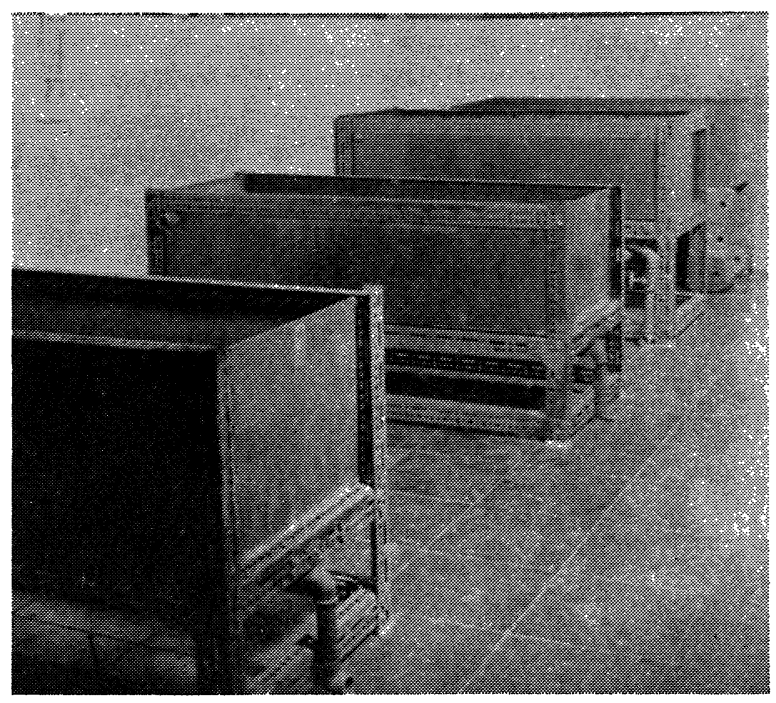

Fig. 6.-Balsas para el curado de probetas.

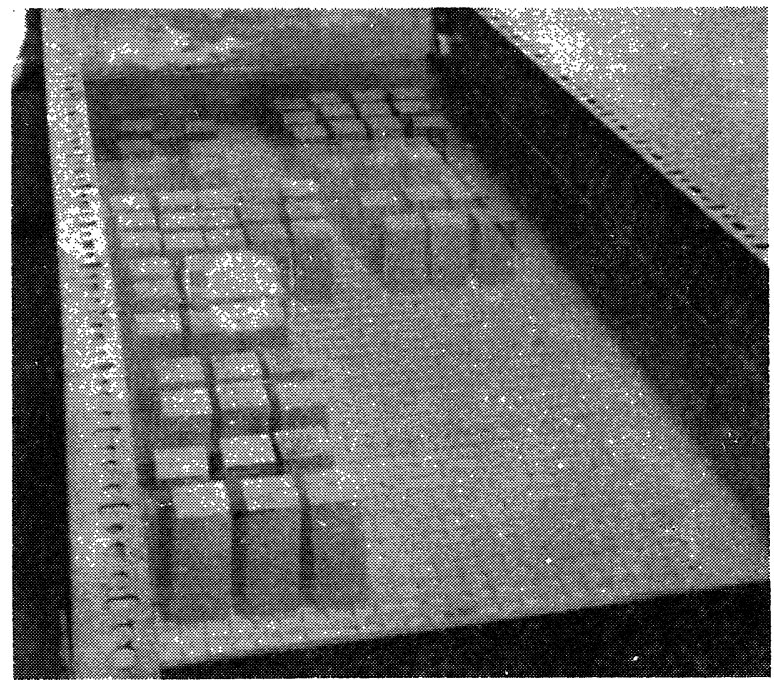

Fig. 7.-Aspecto interior de una balsa de curado de probetas.

que contienen las balsas, de tal manera que entre las probetas exista un espacio libre de 5 a $10 \mathrm{~mm}$, quedando a su vez apoyadas en la rejilla del fondo (Fig. 7); de esta forma se consigue que el agua rodee completamente a la probeta.

En cada balsa, y en caso de utilizar diversos tipos de cemento, sólo se introducen probetas que pertenezcan al mismo tipo.

\section{PROCEDIMIENTO OPERATORIO}

Antes de iniciar las operaciones de control, de una "arena normalizada", se comprueban sus características químicas y físicas, las cuales deberán cumplir con las exigencias mínimas especificadas en la Norma correspondiente. 
Una vez hecha la comprobación, se elige el tipo de cemento y se toman las muestras necesarias de arena, de acuerdo con lo señalado en los apartados 2.3.1. y 2.3.2.

Con el cemento y la arena elegidos se preparan, mensualmente, ocho amasadas de mortero para la "arena de referencia", y otras tantas para la "arena normalizada" que se controla. Dichas amasadas son realizadas de conformidad con las normas correspondientes a los distintos tipos de arena.

Los ensayos de resistencia a compresión, para cada tipo de arena, se hacen por duplicado a las edades de 1, 3, 7 y 28 días.

\subsection{Compactación y moldeo}

Los moldes, una vez aceitados y colocadas las marcas de identificación, se sitúan en la compactadora (Fig. 8), rellenándose en dos veces, dando en cada una de ellas 60 golpes. Inmediatamente se retira el molde de la compactadora, se elimina el exceso de mortero y se alisa la superficie por medio de una regla metálica. A continuación, el molde se traslada al armario de curado, cubriéndolo con una placa de plástico de $10 \mathrm{~mm}$ de espesor (Fig. 8), con objeto de evitar pérdidas por evaporación.

\subsection{Desmoldeo}

El desmoldèo se realiza a las 24 horas de haber efectuado la amasada, poniendo una marca, en cada una de las probetas, de la manera siguiente:

Las de un día con las letras $A, B$ y $C$, teniendo en cuenta que la letra $A$ corresponde a la probeta situada en la parte exterior del molde en el momento de la compactación.

Las probetas correspondientes a las edades de 3,7 y 28 dias se encuentran situadas en distintos lugares dentro de cada uno de los moldes A, B, C (Fig. 8), de acuerdo con el esquema abajo indicado.

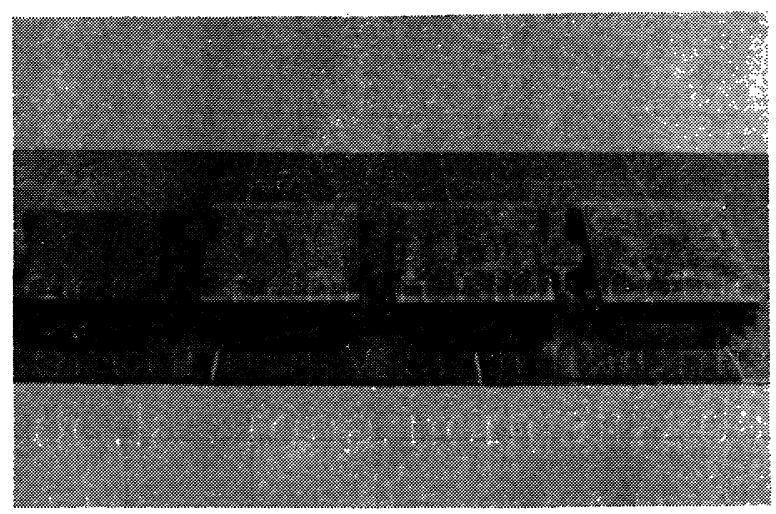

Fig. 8.-Probetas en sus moldes de acero para ser introducidas en la cámara de curado.

Fotos: J. J. López del Amor. IETcc/CSIC.

Con este sistema se pretende conocer, si la compactación es homogénea, evitar las posibles diferencias de compactación y, al mismo tiempo, con sólo mirar la hoja de resultados podemos conocer el lugar que había ocupado la probeta en el molde, puesto que la señalada con el 1 pertenece siempre a la situación en la parte exterior del molde, cuando éste se encuentra colocado en la compactadora.

Desmoldadas las probetas se pesan y, a las 24 horas, se ensayan inmediatamente; el resto se introducen adecuadamente en la balsa de curado, hasta que cumplen la fecha de rotura.

\subsection{Ensayos}

Una vez las probetas hayan cumplido la edad, se determinan las resistencias a flexotracción y compresión.

Los resultados obtenidos, así como la granulometría de arena, tipo de cemento y Norma empleada, figuran en las hojas de resultados, que a tal efecto tiene el Instituto.

\section{RESULTADOS}

Efectuados los ensayos de resistencia a com-

\section{ESQUEMA}

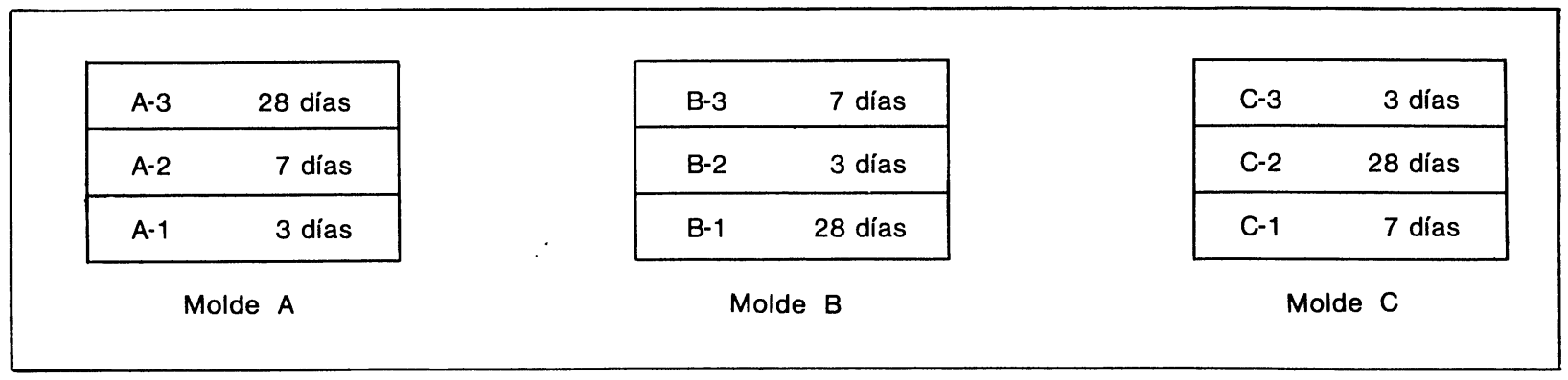


presión de la arena a controlar, se comparan con los obtenidos con el mismo cemento y la "arena de referencia IET/CEN", comprobándose a continuación si los valores cumplen con el mínimo exigido por la Norma CEN-EN196-1.

A título de ejemplo se han entresacado del Libro de Control y Seguimiento de la Arena del Instituto tres tablas de resultados de los ensayos de resistencia, una de cada tipo de arena (IET/CEN, UNE, y RC-75), hojas que se incluyen a continuación.

Si examinamos las tablas de resultados podemos observar que los valores medios de resistencia a compresión, a las distintas edades de las arenas españolas UNE y RC-75, difieren menos del $5 \%$ en relación con la "arena de referencia IET/CEN".

ARENA : IET CEN - Fabricada: Septlembre-1987

Procedimiento operatorio : Norma. CEN-EN 196-1

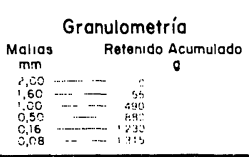

Cemento : P.450 ARI

RESISTENCIAS MECANICAS-FECHA DE MOLDEO 8/9/198

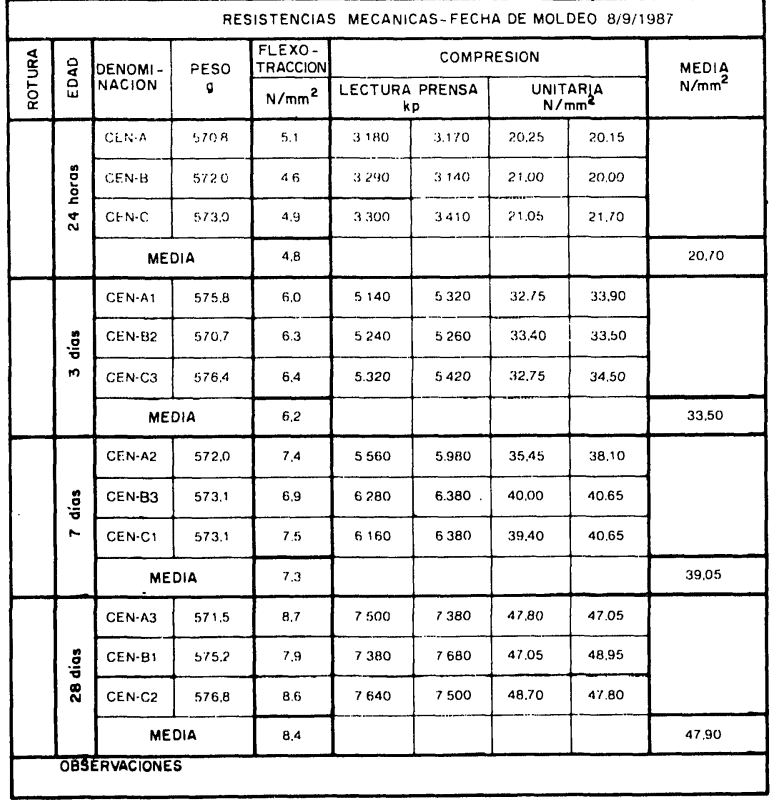

ARENA : U N.E - Fabricada. Jullo-198\%

Procedimiento operatorio : UNE-80101

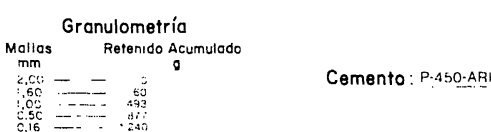

\begin{tabular}{|c|c|c|c|c|c|c|c|c|c|}
\hline \multicolumn{10}{|c|}{ RESISTENCIAS MECANICAS-FECHA DE MOLDEO 14/9/1987 } \\
\hline \multirow{2}{*}{ 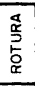 } & \multirow{2}{*}{ ذ̊̀ } & \multirow{2}{*}{$\begin{array}{l}\text { DENOMI- } \\
\text { NACION }\end{array}$} & \multirow{2}{*}{$\begin{array}{c}\text { PESO } \\
g\end{array}$} & \multirow{2}{*}{$\begin{array}{l}\text { FLEXO- } \\
\text { TRACCION } \\
\mathrm{N} / \mathrm{mm}^{2} \\
\end{array}$} & \multicolumn{4}{|c|}{ COMPRESION } & \multirow{2}{*}{$\begin{array}{l}\text { MEDIA } \\
\mathrm{N} / \mathrm{mm}^{2}\end{array}$} \\
\hline & & & & & \multicolumn{2}{|c|}{$\begin{array}{c}\text { LECTURA PRENSA } \\
\text { kD }\end{array}$} & \multicolumn{2}{|c|}{$\begin{array}{l}\text { UNITARIA } \\
\mathrm{N} / \mathrm{mm}^{2}\end{array}$} & \\
\hline & \multirow{4}{*}{$\begin{array}{l}6 \\
E \\
\dot{E} \\
\dot{N}\end{array}$} & LNE-A & 570.4 & $\therefore$ & 3160 & $\$ 140$ & 20.15 & 20.00 & \\
\hline & & UNE B & $5 \% 5.5$ & .5 & 3120 & 3200 & 19.85 & 20.40 & \\
\hline & & UNI-C & $36: 4$ & $4 . \tau$ & 3.180 & 3200 & 20.25 & 20.40 & \\
\hline & & \multicolumn{2}{|c|}{ MEDIA } & 4.5 & & & & & 20,20 \\
\hline & \multirow{4}{*}{$\begin{array}{l}.6 \\
\text { m } \\
m\end{array}$} & UNE-AT & $: 5 / 4$ & 6.8 & 5620 & 5.560 & 35.80 & 35.45 & \\
\hline & & UNF-Ez & soo. & 64 & 5300 & 5260 & 33.50 & 33.50 & \\
\hline & & UNECG? & 670,8 & 66 & 5580 & 5460 & 35.60 & 34.80 & \\
\hline & & \multicolumn{2}{|c|}{ MEDIA } & 6.6 & & & & & 34.80 \\
\hline & \multirow{4}{*}{$\begin{array}{l}\frac{6}{6} \\
\text { r }\end{array}$} & UNE.A2 & $567 . t$ & 7.2 & 6380 & 6400 & 40.70 & 40.80 & \\
\hline & & UNF-B33 & 5676 & 7.6 & 6030 & 6140 & 38.40 & 39.15 & \\
\hline & & UNE-C1 & 574.3 & 69 & 6280 & 5.160 & 40.05 & 39.30 & \\
\hline & & \multicolumn{2}{|c|}{ MEDIA } & 72 & & & & & 39.70 \\
\hline & \multirow{4}{*}{ 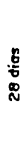 } & UNE-A3 & 569.1 & 86 & 7480 & 7540 & 47.70 & 48.05 & \\
\hline & & UNE-B1 & 367.8 & 8.2 & 7560 & 7580 & 48.20 & 48,30 & \\
\hline & & UNF-C2 & $b / 5.0$ & 8.8 & 7.520 & 7600 & 41.90 & 48.45 & \\
\hline & & \multicolumn{2}{|c|}{ MEDIA } & 8.5 & & & & & 48.10 \\
\hline
\end{tabular}

ARENA : RC-75 - Fabricada: Septiembre-1987.

Procedimiento operatorio : Pleggo - $\mathrm{AC}-15$

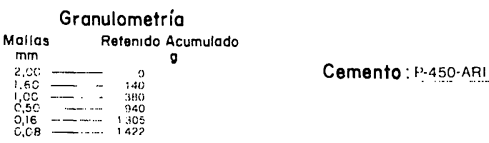

\begin{tabular}{|c|c|c|c|c|c|c|c|c|c|}
\hline \multicolumn{10}{|c|}{ RESISTENCIAS MECANICAS-FECHA DE MOLDEO 15/9/1987 } \\
\hline \multirow{2}{*}{ 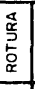 } & \multirow{2}{*}{ 总 } & \multirow{2}{*}{$\begin{array}{l}\text { DENOMI- } \\
\text { NACION }\end{array}$} & \multirow{2}{*}{$\begin{array}{c}\text { PESO } \\
\mathbb{g}\end{array}$} & $\begin{array}{l}\text { FLEXO- } \\
\text { TRACCION }\end{array}$ & \multicolumn{4}{|c|}{ COMPRESION } & \multirow{2}{*}{$\begin{array}{l}\mathrm{MEDIA} \\
\mathrm{N} / \mathrm{mm} \mathrm{m}^{\mathrm{E}}\end{array}$} \\
\hline & & & & $\mathrm{N} / \mathrm{mm}^{2}$ & \multicolumn{2}{|c|}{$\begin{array}{l}\text { LECTURA PRENSA } \\
\text { KD }\end{array}$} & \multicolumn{2}{|c|}{$\begin{array}{l}\text { UNITARIA } \\
\mathrm{N} / \mathrm{mm}^{2}\end{array}$} & \\
\hline & \multirow{4}{*}{$\begin{array}{l}\mathbf{E} \\
\mathbf{5} \\
\mathbf{2} \\
\mathbf{N}\end{array}$} & $\mathrm{BC}-7 ;,-4$ & 578.6 & $4 . i$ & 3220 & 3.200 & 20.50 & 20.40 & \\
\hline & & $\mathrm{BC} \cdot 75 . \mathrm{B}$ & $5: 16$ & 4.6 & 3340 & 3400 & 21.30 & 21,65 & \\
\hline & & $\mathrm{RC}-\sim 5 \mathrm{C}$ & 578.4 & 4.8 & 3.420 & 3.380 & 21.80 & 21,50 & \\
\hline & & \multicolumn{2}{|c|}{ MEDIA } & 4.7 & & & , & & 21.20 \\
\hline & \multirow{4}{*}{$\begin{array}{l}\underset{6}{6} \\
\text { 雨 }\end{array}$} & $\mathrm{RC}-7 \mathrm{5}-\mathrm{A} 1$ & 580.1 & 6.2 & 5200 & 5.400 & 33.15 & 34,40 & \\
\hline & & $\mathrm{AC}-75-82$ & 578.2 & 6.4 & 5.320 & 5.300 & 33,90 & 33.80 & \\
\hline & & $R C-75 \cdot C 3$ & 579.6 & 6.2 & 5280 & 5.360 & 33,65 & 34.15 & \\
\hline & & \multicolumn{2}{|c|}{ MEDIA } & 6.3 & & & & & 33.85 \\
\hline & \multirow{4}{*}{ 年 } & RC- $75-A^{2} 2$ & 577.5 & 1.3 & 5.800 & 6180 & 37,00 & 39.40 & \\
\hline & & $\mathrm{AC} \cdot 75 \cdot \mathrm{B3}$ & 579.4 & 1.5 & 6100 & 6.220 & 38.90 & 39.65 & \\
\hline & & $\mathrm{RC} \cdot 75 . \mathrm{Cl}$ & 578.8 & 7.4 & 6.360 & 6.340 & 40.55 & 40.40 & \\
\hline & & \multicolumn{2}{|c|}{ MEDIA } & 1.4 & & & & & 39,30 \\
\hline & \multirow{4}{*}{ 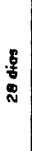 } & $\mathrm{RC}-75 \cdot \mathrm{A} 3$ & 575.7 & 8.4 & 7.600 & 7650 & 48.45 & 48.75 & \\
\hline & & AC. 75 - B1 & 578.0 & 8.7 & 7540 & 7.540 & 48.05 & 48.05 & \\
\hline & & $\mathrm{RC}-75 \cdot \mathrm{C} ?$ & 577.5 & 8.5 & 7.700 & 7640 & 49.10 & 48,70 & \\
\hline & & \multicolumn{2}{|c|}{ MEDIA } & 8.5 & & & & & 48.50 \\
\hline
\end{tabular}

MATERIALES DE CONSTRUCCION, Vol. 38, n.॰ 210, abril/mayo/junio 1988 